\title{
Superior Intercostal Vein
}

National Cancer Institute

\section{Source}

National Cancer Institute. Superior Intercostal Vein. NCI Thesaurus. Code C33680.

Either of two veins (left or right) that drain the second, third, and fourth intercostal

spaces. The right superior intercostal vein drains into the right azygos vein while the left superior intercostal vein drains into the left innominate vein. 